\title{
Brachial Plexus Neuropraxia after CT-Guided Radiofrequency Ablation in a Patient with a Narrowed Costoclavicular Space
}

\section{J.P. Lew, A. Zeng, Y.Y. Ong Singapore General Hospital}

Brachial plexus neuropraxia during anaesthesia has been reported in multiple different surgeries and is caused by the stretching and compression of the plexus secondary to positioning. Anatomical abnormalities of the thoracic outlet with which the plexus runs through as a contributory aggravating cause of such injury has however been less discussed.

We describe a case of brachial plexus neuropraxia after radiofrequency abalation in a patient with a previously unknown narrowed costoclavicular space and discuss ways of examining and identifying patients with thoracic outlet narrowing which can increase their risk of postoperative neuropraxia.

\section{Introduction}

The patient is a 54 year old gentleman, $169 \mathrm{~cm} 62 \mathrm{~kg}$, with a history of diabetes mellitus, hypertension, hepatitis $\mathrm{C}$ complicated with Childs A liver cirrhosis on Peginterferon alfa-2a and ribavirin. He was diagnosed with primary hepatocellular carcinoma in hepatic segment $6 / 7$. The lesion measured $3.1 \times 2.6 \times 2.7 \mathrm{~cm}$ and was indenting the retro hepatic inferior vena cava. He was scheduled for CT-guided radiofrequency ablation of the lesion.

The procedure was done in half left lateral position (body tilted 15 degrees to the left) on a CT table with both arms raised above his head i.e. elbows flexed, shoulders abducted and externally rotated, wrists pronated, and head in a neutral position. This position allows the proceduralist access and avoids CT image degradation from streak artifacts. All pressure points were protected during the procedure. The procedure was done under deep sedation for the first 1.5 hours; with a target controlled infusion of propofol titrated to sedation score. General anaesthesia had to be instituted for the last hour of the procedure as the patient was feeling uncomfortable and found it difficult to cooperate with the radiologist. The general anaesthesia was conducted with a supraglottic airway, spontaneous ventilation and a total intravenous anaesthesia technique. The patient remained haemodynamically stable throughout the procedure with no episodes of hypoxia. The entire duration was 2.5 hours.

The patient complained about right upper limb weakness and numbness a few hours after the procedure, affecting mainly the $\mathrm{C} 5$ and $\mathrm{C} 6$ root distributions. Power on shoulder abduction (C5) was 2/5, elbow flexion (C6) was 2/5, elbow extension (C7) was $3 / 5$ and wrist extension (C6) $3+/ 5$. Sensory loss was noted over the C5-C7 dermatomes. CT brain to rule out a stroke was normal. MRI brachial plexus done the next day showed abnormal enhancement along the right brachial plexus involving the divisions and trunks segments from the costoclavicular space to the interscalene triangle. His costoclavicular space was noted to be narrowed. See figure 1 and 2. The patient was given an arm sling and sessions with an occupational therapist. He was also followed up by a neurologist. Nerve conduction studies done 1 month later was inconclusive for right brachial plexopathy. Fortunately for the patient, he recovered $90 \%$ of his right upper limb sensation and strength 3 months after the injury. Power on shoulder abduction was $4+/ 5$, elbow flexion was $4+/ 5$, elbow extension was $5 / 5$ and wrist extension $4+/ 5$.

\section{Discussion}

There are many factors to consider in the development of brachial plexus neuropraxia. This includes the anatomy of the thoracic outlet as well as the anatomy of the brachial plexus. The plexus lies relatively superficial during its course down the neck and into the axilla. It also has 2 fixation points, the vertebra and prevertebral fascia proximally and the axillary fascia distally, thus making it vulnerable to injury from compression, stretching and kinking. Stretching of the brachial plexus results in minute intraneural capillary rupture with formation of small hematomas, thus resulting in compression and thus ischemia of the nerve bundles. Nerve injuries can be classified based on the nerve pathology according to Seddon's classification. Of interest to anaesthesia; neurapraxia occurs when there is damaged myelin with an intact axon. Recovery usually occurs in weeks to months and the prognosis is generally good.

Apart from anatomical and positional factors there are also multiple patient and surgical factors associated with peripheral nerve injury occurring with anaesthesia. These include intraoperative hypotension, hypovolemia and hypothermia, pre-existing neuropathy including vitamin B12 deficiency, diabetes mellitus, alcoholism and smoking. Surgical factors include long operative time and type of surgery, especially those where there is possible direct injury to nerves.

Identification of risk factors is the key to prevention of such injuries. Patients with a narrowed thoracic outlet can have symptoms and signs of vascular or neuropathic compromise. Provocative clinical testing for thoracic outlet syndrome has been reported to display high rates of false positive but are relatively simple to do and noninvasive. This includes elevated arm stress test or the costoclavicular maneuvre. Extra caution should be taken if thoracic outlet narrowing is suspected.

References

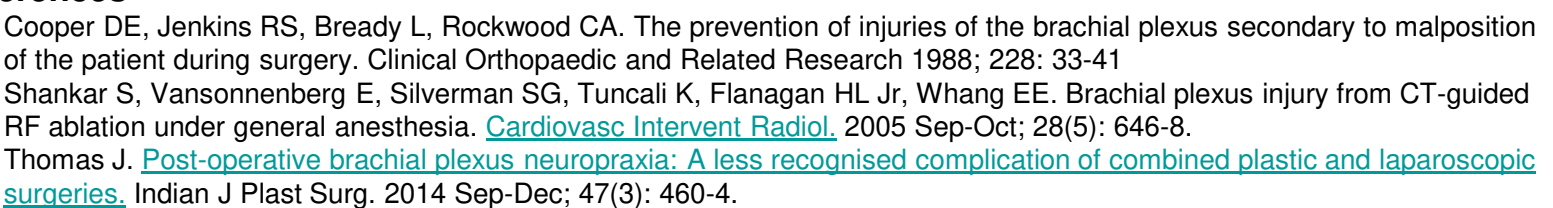

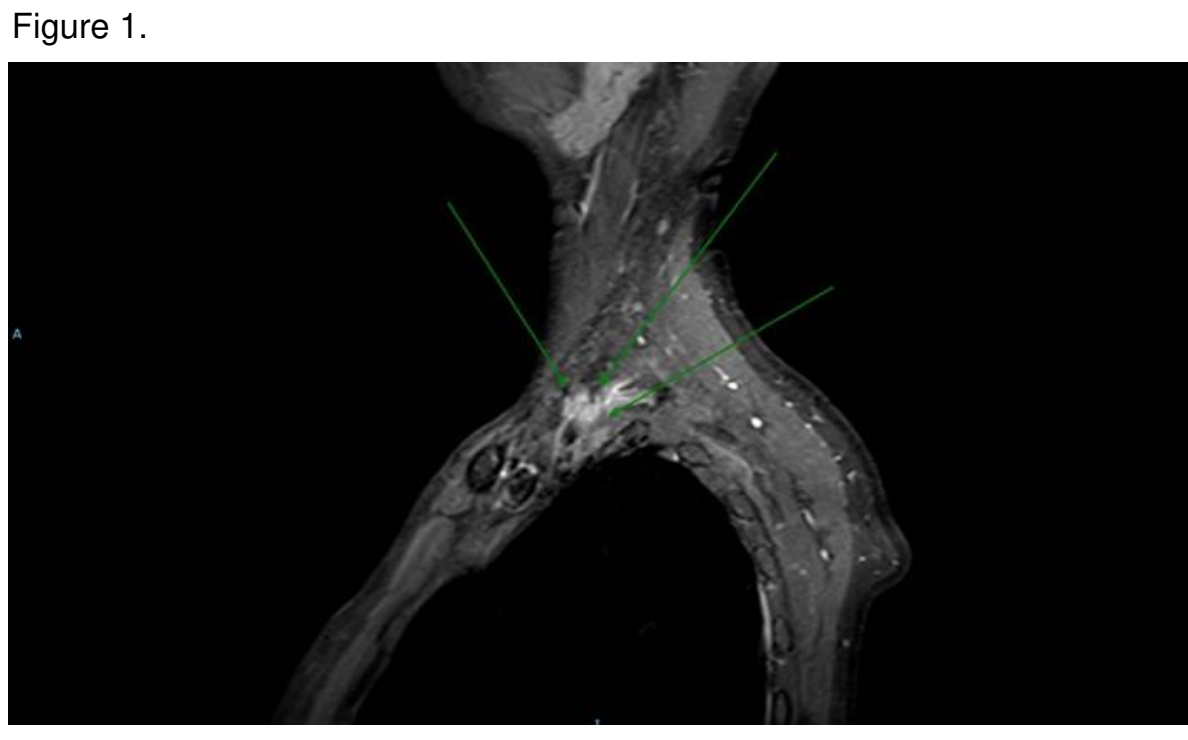

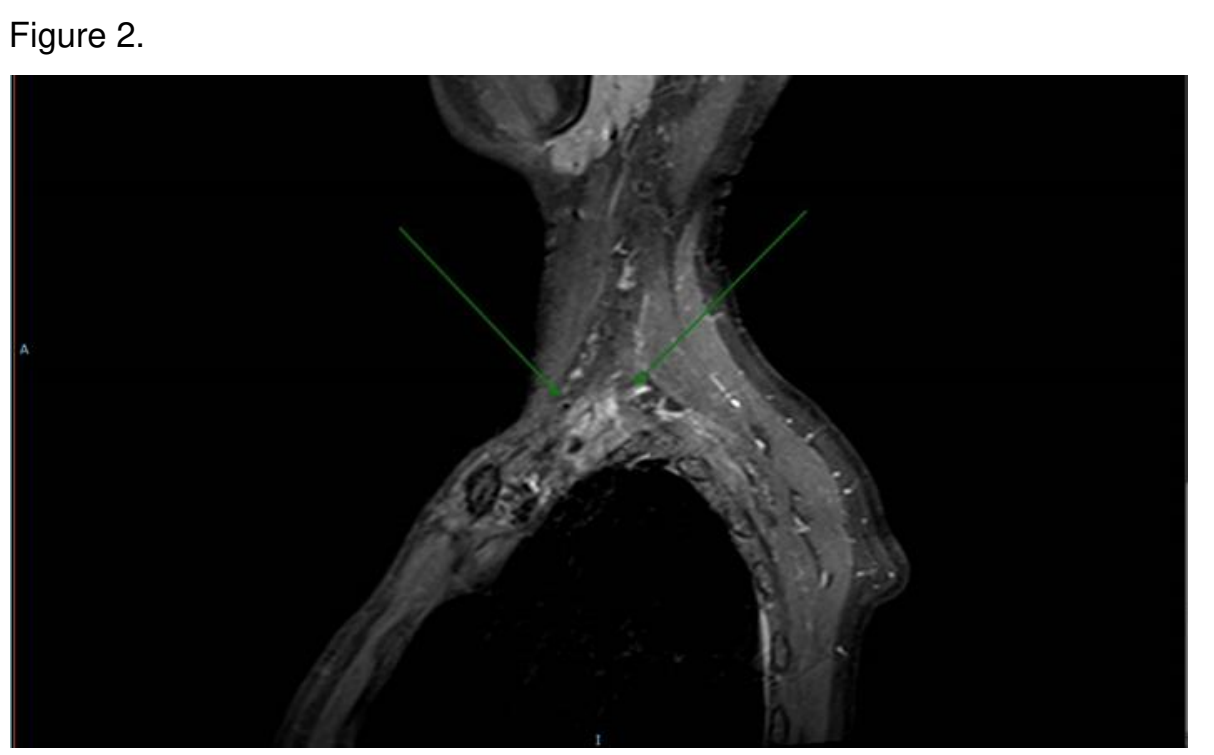

General anaesthesia as well as the use of muscle relaxants increases the risk of brachial plexus neuropraxia as they prevent the patient from correcting nonphysiological positions. If a sedation technique is used, the patient must be instructed to inform the operator if he should have any discomfort in his arm

Avoiding an arm above head position is useful in reducing stretch on the brachial plexus. For CT-guided ablation, Shankar et al recommends positioning the arms crossed across the chest and slightly raised with padding from the chest. This succeeds in giving the operator access and also good CT images of the liver while reducing risk of brachial plexus neuropraxia. If an arm across chest position is not possible, frequent arm position changes and restricting the arm above head position for only vital portions of the procedure will also help. Intraoperative monitoring with somatic sensory evoked potentials has also been suggested and might be helpful.

\section{Conclusion}

In a patient suspected with brachial plexus neuropraxia, a thorough neurological examination should be done. Postoperative paresthesia, pain, numbness and weakness should be treated seriously and promptly. All motor or sensory loss should be documented. There might be a need to exclude stroke or a cervical spine injury especially if there are accompanying lower limb neurological signs. Management of the injury should involve a multidisciplinary team of neurologist, occupational therapist and physiotherapist. A magnetic resonance imaging of the brachial plexus can be done. Nerve conduction studies and electromyography (EMG) may assist in diagnosing brachial plexus neuropraxia, as well as determining its age and severity. Typical EMG denervation changes in the upper limb appear 2-3 weeks after the injury. Early EMG helps to investigate for a pre-existing neuropathy.

Treatment of brachial plexus neuropraxia consists of mainly occupational therapy and physiotherapy. Arm exercises and muscle training can assist in its recovery by keeping the joints supple. Pain is controlled by simple analgesics and antiepileptic agents like gabapentin have been used for related neuropathic pain

The prognosis of brachial plexus neuropraxia is generally good with most recovering fully by 20 weeks. 